\title{
Elemental analysis of Oxygenated Organic Coating on Black Carbon Particles using a Soot-Particle Aerosol Mass Spectrometer
}

\author{
Mutian $\mathrm{Ma}^{1}$, Laura-Hélèna Rivellini ${ }^{2}$, YuXi Cui ${ }^{1}$, Megan D. Willis ${ }^{3}$, Rio Wilkie ${ }^{4}$, Jonathan P. D. Abbatt ${ }^{4}$, Manjula R. \\ Canagaratna $^{5}$, Junfeng Wang ${ }^{6}$, Xinlei Ge ${ }^{6}$, Alex K.Y. Lee ${ }^{1,2}$ \\ ${ }^{1}$ Department of Civil and Environmental Engineering, National University of Singapore, Singapore \\ ${ }^{2}$ NUS Environmental Research Institute, National University of Singapore, Singapore \\ ${ }^{3}$ Lawrence Berkeley National Lab, Chemical Sciences Division, Berkeley, CA, USA \\ ${ }^{4}$ Department of Chemistry, University of Toronto, Toronto, ON, Canada \\ ${ }^{5}$ Aerodyne Research, Inc., Billerica, MA, USA \\ ${ }^{6}$ School of Environmental Science and Engineering, Nanjing University of Information Science and Technology, \\ Nanjing, China
}

Correspondence to: Alex K. Y. Lee (ceelkya@nus.edu.sg) 
Table S1. Summary of elemental ratios and time series correlation of PMF factors based on co-located measurements in Beijing summer (Xie et al., 2019a; Xu et al., 2019), Beijing winter (Wang et al., 2019; Xie et al., 2019b), California Research at the Nexus of Air Quality and Climate Change (CalNex) 2010 campaign (Massoli et al., 2015), Fontana, CA (Chen et al., 2018; Lee et al., 2017), and Tibet (Wang et al., 2017; Xu et al., 2018).

\begin{tabular}{|c|c|c|c|c|c|c|c|c|c|}
\hline \multirow{2}{*}{$\begin{array}{l}\text { Location / } \\
\text { Campaign }\end{array}$} & \multicolumn{4}{|c|}{ HR-ToF-AMS } & \multicolumn{4}{|c|}{ SP-AMS LV scheme ${ }^{\#}$} & \multirow[b]{2}{*}{$\mathrm{R}$} \\
\hline & Study & $\begin{array}{l}\text { PMF } \\
\text { factor }\end{array}$ & $\mathrm{H}: \mathrm{C}$ & $\mathrm{O}: \mathrm{C}$ & Study & $\begin{array}{l}\text { PMF } \\
\text { factor }\end{array}$ & $\mathrm{H}: \mathrm{C}$ & $\mathrm{O}: \mathrm{C}$ & \\
\hline Beijing summer & $\begin{array}{l}\text { Xie et al. } \\
(2019 a)\end{array}$ & $\begin{array}{l}\text { LO- } \\
\text { OOA }\end{array}$ & 1.34 & 0.49 & $\begin{array}{l}\text { Xu et al. } \\
(2019)\end{array}$ & $\begin{array}{l}\mathrm{LO}- \\
\mathrm{OOA}\end{array}$ & 1.6 & 0.28 & 0.88 \\
\hline Beijing winter & $\begin{array}{l}\text { Xie et al. } \\
(2019 b)\end{array}$ & $\begin{array}{l}\text { LO- } \\
\text { OOA }\end{array}$ & 1.61 & 0.6 & $\begin{array}{l}\text { Wang et al. } \\
\text { (2019) }\end{array}$ & OOA1 & 1.55 & 0.37 & 0.92 \\
\hline Beijing winter & $\begin{array}{l}\text { Xie et al. } \\
(2019 b)\end{array}$ & $\begin{array}{l}\text { MO- } \\
\text { OOA }\end{array}$ & 1.36 & 0.97 & $\begin{array}{l}\text { Wang et al. } \\
\text { (2019) }\end{array}$ & OOA2 & 1.57 & 1.23 & 0.97 \\
\hline CalNex & $\begin{array}{l}\text { Massoli et al. } \\
\text { (2015) }\end{array}$ & $\begin{array}{l}\text { SV- } \\
\text { OOA }\end{array}$ & 1.58 & 0.54 & $\begin{array}{l}\text { Massoli et al. } \\
\text { (2015) }\end{array}$ & $\begin{array}{l}\text { SV- } \\
\text { OOA }\end{array}$ & 1.83 & 0.45 & 0.92 \\
\hline CalNex & $\begin{array}{l}\text { Massoli et al. } \\
\text { (2015) }\end{array}$ & $\begin{array}{l}\text { LV- } \\
\text { OOA }\end{array}$ & 1.2 & 1.4 & $\begin{array}{l}\text { Massoli et al. } \\
\text { (2015) }\end{array}$ & $\begin{array}{l}\text { LV- } \\
\text { OOA }\end{array}$ & 1.4 & 1.16 & 0.84 \\
\hline Fontana, CA & $\begin{array}{l}\text { Chen et al. } \\
\text { (2018) }\end{array}$ & VOOA & 0.78 & 1.4 & $\begin{array}{l}\text { Lee et al. } \\
(2017)\end{array}$ & OOA2 & 1.63 & 0.63 & 0.76 \\
\hline Tibet & $\begin{array}{c}\text { Xu et al. } \\
(2018)\end{array}$ & $\begin{array}{l}\text { MO- } \\
\text { OOA }\end{array}$ & 1.04 & 0.96 & $\begin{array}{l}\text { Wang et al. } \\
\text { (2017) }\end{array}$ & BBOA & 1.48 & 0.51 & 0.96 \\
\hline
\end{tabular}

\# The inter-conversion factors that were determined in this work, were applied for calculating H:C and O:C ratios. 
Table S2. Summary of H:C, O:C and. $\mathrm{OS}_{\mathrm{c}}$ determined by the SP-AMS 1

\begin{tabular}{|c|c|c|c|c|c|c|c|c|c|c|c|}
\hline \multirow[b]{2}{*}{ Class } & \multirow[b]{2}{*}{ Species } & \multirow[b]{2}{*}{ Formula } & \multicolumn{3}{|c|}{ True value } & \multicolumn{3}{|c|}{ Tungsten vaporizer } & \multicolumn{3}{|c|}{ Laser vaporizer } \\
\hline & & & H:C & O:C & OSc & H:C & O:C & OSc & H:C & O:C & OSc \\
\hline Multifunctional & Citric acid & $\mathrm{C}_{6} \mathrm{H}_{8} \mathrm{O}_{7}$ & 1.33 & 1.17 & 1.00 & 1.42 & 0.93 & 0.43 & 1.66 & 0.72 & -0.21 \\
\hline Multifunctional & Glycolic acid & $\mathrm{C}_{2} \mathrm{H}_{4} \mathrm{O}_{3}$ & 2.00 & 1.50 & 1.00 & 1.86 & 0.78 & -0.31 & 1.71 & 0.53 & -0.64 \\
\hline Multifunctional & Malic Acid & $\mathrm{C}_{4} \mathrm{H}_{6} \mathrm{O}_{5}$ & 1.50 & 1.25 & 1.00 & 1.55 & 1.00 & 0.44 & 1.63 & 1.00 & 0.37 \\
\hline Multifunctional & Tartaric acid & $\mathrm{C}_{4} \mathrm{H}_{6} \mathrm{O}_{6}$ & 1.50 & 1.50 & 1.50 & 1.48 & 1.34 & 1.20 & 1.63 & 1.52 & 1.40 \\
\hline Diacids & Adipic Acid & $\mathrm{C}_{6} \mathrm{H}_{10} \mathrm{O}_{4}$ & 1.67 & 0.67 & -0.33 & 1.71 & 0.60 & -0.51 & 1.71 & 0.46 & -0.79 \\
\hline Diacids & Azelaic Acid & $\mathrm{C}_{9} \mathrm{H}_{16} \mathrm{O}_{4}$ & 1.78 & 0.44 & -0.89 & 1.65 & 0.29 & -1.07 & 1.90 & 0.35 & -1.19 \\
\hline Diacids & Glutaric acid & $\mathrm{C}_{5} \mathrm{H}_{8} \mathrm{O}_{4}$ & 1.60 & 0.80 & 0 & 1.64 & 0.44 & -0.76 & 1.66 & 0.47 & -0.71 \\
\hline Diacids & Malonic acid & $\mathrm{C}_{3} \mathrm{H}_{4} \mathrm{O}_{4}$ & 1.33 & 1.33 & 1.33 & 1.36 & 1.12 & 0.87 & 1.61 & 1.10 & 0.59 \\
\hline Diacids & Oxalic acid & $\mathrm{C}_{2} \mathrm{H}_{2} \mathrm{O}_{4}$ & 1.00 & 2.00 & 3.00 & 1.13 & 1.91 & 2.68 & 1.36 & 1.72 & 2.09 \\
\hline Diacids & Phthalic acid & $\mathrm{C}_{8} \mathrm{H}_{6} \mathrm{O}_{4}$ & 0.75 & 0.50 & 0.25 & 0.84 & 0.26 & -0.32 & 0.95 & 0.25 & -0.46 \\
\hline Diacids & Pimelic acid & $\mathrm{C}_{7} \mathrm{H}_{12} \mathrm{O}_{4}$ & 1.71 & 0.57 & -0.57 & 1.66 & 0.42 & -0.82 & 1.83 & 0.41 & -1.02 \\
\hline Diacids & Suberic acid & $\mathrm{C}_{8} \mathrm{H}_{14} \mathrm{O}_{4}$ & 1.75 & 0.50 & -0.75 & 1.58 & 0.33 & -0.93 & 1.85 & 0.40 & -1.06 \\
\hline Diacids & Succinic acid & $\mathrm{C}_{4} \mathrm{H}_{6} \mathrm{O}_{4}$ & 1.50 & 1.00 & 0.50 & 1.72 & 0.77 & -0.18 & 1.48 & 0.83 & 0.17 \\
\hline Alcohols & Arabitol & $\mathrm{C}_{5} \mathrm{H}_{12} \mathrm{O}_{5}$ & 2.4 & 1.00 & -0.4 & 1.59 & 0.83 & 0.06 & 2.13 & 0.81 & -0.51 \\
\hline Alcohols & Glucose & $\mathrm{C}_{6} \mathrm{H}_{12} \mathrm{O}_{6}$ & 2.00 & 1.00 & 0 & 2.01 & 1.01 & 0.02 & 2.09 & 1.00 & -0.10 \\
\hline Alcohols & Levoglucosan & $\mathrm{C}_{6} \mathrm{H}_{10} \mathrm{O}_{5}$ & 1.67 & 0.83 & 0 & 1.85 & 0.77 & -0.31 & 2.06 & 0.86 & -0.33 \\
\hline Alcohols & Sucrose & $\mathrm{C}_{12} \mathrm{H}_{22} \mathrm{O}_{11}$ & 1.83 & 0.92 & 0 & 1.90 & 0.900 & -0.10 & 1.99 & 0.83 & -0.32 \\
\hline Alcohols & Xylitol & $\mathrm{C}_{5} \mathrm{H}_{12} \mathrm{O}_{5}$ & 2.40 & 1.00 & -0.4 & 1.92 & 0.78 & -0.37 & 2.21 & 0.90 & -0.41 \\
\hline
\end{tabular}


Table S3. Summary of H:C, O:C and. $\mathrm{OS}_{\mathrm{c}}$ determined by the SP-AMS 2

\begin{tabular}{|c|c|c|c|c|c|c|c|c|c|c|c|}
\hline \multirow[b]{2}{*}{ Species } & \multirow[b]{2}{*}{ Class } & \multirow[b]{2}{*}{ Formula } & \multicolumn{3}{|c|}{ True value } & \multicolumn{3}{|c|}{ Tungsten vaporizer } & \multicolumn{3}{|c|}{ Laser vaporizer } \\
\hline & & & H:C & O:C & OSc & H:C & O:C & OSc & H:C & O:C & OSc \\
\hline $\begin{array}{l}\text { Cis-Pinonic } \\
\text { acid }\end{array}$ & Multifunctional & $\mathrm{C}_{10} \mathrm{H}_{16} \mathrm{O}_{3}$ & 1.60 & 0.30 & -1.00 & 1.69 & 0.25 & -1.19 & 0.48 & 1.30 & -0.34 \\
\hline Citric acid & Multifunctional & $\mathrm{C}_{6} \mathrm{H}_{8} \mathrm{O}_{7}$ & 1.33 & 1.17 & 1.00 & 1.40 & 1.00 & 0.59 & 0.94 & 1.39 & 0.49 \\
\hline Glutamic acid & Multifunctional & $\mathrm{C}_{5} \mathrm{H}_{9} \mathrm{NO}_{4}$ & 1.80 & 0.80 & -0.2 & & NA & & 0.29 & 2.03 & -1.45 \\
\hline Glycolic acid & Multifunctional & $\mathrm{C}_{2} \mathrm{H}_{4} \mathrm{O}_{3}$ & 2.00 & 1.50 & 1.00 & 1.45 & 1.24 & 1.04 & 0.65 & 1.56 & -0.26 \\
\hline Levulinic acid & Multifunctional & $\mathrm{C}_{5} \mathrm{H}_{8} \mathrm{O}_{3}$ & 1.60 & 0.60 & -0.4 & & NA & & 1.00 & 1.81 & 0.19 \\
\hline Malic acid & Multifunctional & $\mathrm{C}_{4} \mathrm{H}_{6} \mathrm{O}_{5}$ & 1.50 & 1.25 & 1.00 & 1.56 & 1.10 & 0.64 & 0.60 & 1.55 & -0.35 \\
\hline Pyruvic acid & Multifunctional & $\mathrm{C}_{3} \mathrm{H}_{4} \mathrm{O}_{3}$ & 1.33 & 1.00 & 0.67 & 1.32 & 0.74 & 0.15 & 1.52 & 1.94 & 1.10 \\
\hline Tartaric acid & Multifunctional & $\mathrm{C}_{4} \mathrm{H}_{6} \mathrm{O}_{6}$ & 1.50 & 1.50 & 1.5 & 1.60 & 1.74 & 1.88 & 0.09 & 2.30 & -2.12 \\
\hline Adipic acid & Diacids & $\mathrm{C}_{6} \mathrm{H}_{10} \mathrm{O}_{4}$ & 1.67 & 0.67 & -0.33 & 1.60 & 0.43 & -0.74 & 0.46 & 1.87 & -0.95 \\
\hline Azelaic acid & Diacids & $\mathrm{C}_{9} \mathrm{H}_{16} \mathrm{O}_{4}$ & 1.78 & 0.44 & -0.89 & 1.62 & 0.32 & -0.99 & 0.32 & 1.94 & -1.30 \\
\hline Glutaric acid & Diacids & $\mathrm{C}_{5} \mathrm{H}_{8} \mathrm{O}_{4}$ & 1.6 & 0.80 & 0 & 1.46 & 0.57 & -0.33 & 0.56 & 1.80 & -0.68 \\
\hline Maleic acid & Diacids & $\mathrm{C}_{4} \mathrm{H}_{4} \mathrm{O}_{4}$ & 1.00 & 1.00 & 1.00 & 1.56 & 1.10 & 0.64 & 0.65 & 1.34 & -0.04 \\
\hline Malonic acid & Diacids & $\mathrm{C}_{3} \mathrm{H}_{4} \mathrm{O}_{4}$ & 1.33 & 1.33 & 1.33 & 1.58 & 1.09 & 0.59 & 1.13 & 1.83 & 0.43 \\
\hline Oxalic acid & Diacids & $\mathrm{C}_{2} \mathrm{H}_{2} \mathrm{O}_{4}$ & 1.00 & 2.00 & 3.00 & 0.92 & 2.42 & 3.92 & 1.68 & 1.34 & 2.02 \\
\hline Succinic acid & Diacids & $\mathrm{C}_{4} \mathrm{H}_{6} \mathrm{O}_{4}$ & 1.50 & 1.00 & 0.50 & 1.64 & 0.50 & -0.64 & 0.55 & 1.89 & -0.79 \\
\hline $\begin{array}{l}\text { Tricarballylic } \\
\text { acid }\end{array}$ & Polyacids & $\mathrm{C}_{6} \mathrm{H}_{8} \mathrm{O}_{6}$ & 1.33 & 1.00 & 0.67 & 1.30 & 0.65 & -0.01 & 0.56 & 1.57 & -0.45 \\
\hline $\begin{array}{l}1,5- \\
\text { Pentanediol }\end{array}$ & Alcohols & $\mathrm{C}_{5} \mathrm{H}_{12} \mathrm{O}_{2}$ & 2.4 & 0.40 & -1.6 & & NA & & 0.44 & 1.70 & -0.82 \\
\hline Dextrose & Alcohols & $\mathrm{C}_{6} \mathrm{H}_{12} \mathrm{O}_{6}$ & 2.00 & 1.00 & 0 & & NA & & 0.87 & 1.87 & -0.13 \\
\hline Phenol & Alcohols & $\mathrm{C}_{6} \mathrm{H}_{6} \mathrm{O}$ & 1.00 & 0.17 & -0.67 & & NA & & 0.62 & 1.81 & -0.57 \\
\hline $\begin{array}{l}\text { Bis(2- } \\
\text { ethylhexyl) } \\
\text { Sebacate }\end{array}$ & Esters & $\mathrm{C}_{26} \mathrm{H}_{50} \mathrm{O}_{4}$ & 1.92 & 0.15 & -1.62 & & NA & & 0.1 & 2.23 & -2.03 \\
\hline
\end{tabular}


Table S4. Summary of H:C, O:C and. $\mathrm{OS}_{\mathrm{c}}$ determined by the SP-AMS 3

\begin{tabular}{llllllllllll}
\hline & & & \multicolumn{3}{c}{ True value } & \multicolumn{3}{c}{ Tungsten vaporizer } & \multicolumn{3}{c}{ Laser vaporizer } \\
\hline Species & Class & Formula & H:C & O:C & OSc & H:C & O:C & OSc & H:C & O:C & OSc \\
\hline Cis-Pinonic Acid & Multifunctional & $\mathrm{C}_{10} \mathrm{H}_{16} \mathrm{O}_{3}$ & 1.60 & 0.30 & -1.00 & 1.43 & 0.22 & -0.99 & 1.53 & 0.19 & -1.15 \\
Citric acid & Multifunctional & $\mathrm{C}_{6} \mathrm{H}_{8} \mathrm{O}_{7}$ & 1.33 & 1.17 & 1.00 & 1.18 & 1.03 & 0.88 & 1.19 & 0.86 & 0.52 \\
Ketoglutaric Acid & Multifunctional & $\mathrm{C}_{5} \mathrm{H}_{6} \mathrm{O}_{5}$ & 1.20 & 1.00 & 0.80 & 1.20 & 0.84 & 0.48 & 1.44 & 0.54 & -0.36 \\
Ketopimelic acid & Multifunctional & $\mathrm{C}_{7} \mathrm{H}_{10} \mathrm{O}_{5}$ & 1.43 & 0.71 & 0 & 1.10 & 0.57 & 0.04 & 1.39 & 0.42 & -0.55 \\
Tartaric acid & Multifunctional & $\mathrm{C}_{4} \mathrm{H}_{6} \mathrm{O}_{6}$ & 1.50 & 1.50 & 1.50 & 1.40 & 1.51 & 1.62 & 1.66 & 1.55 & 1.43 \\
\hline Azelaic Acid & Diacids & $\mathrm{C}_{9} \mathrm{H}_{16} \mathrm{O}_{4}$ & 1.78 & 0.44 & -0.89 & 1.34 & 0.28 & -0.78 & 1.59 & 0.23 & -1.13 \\
Glutaric acid & Diacids & $\mathrm{C}_{5} \mathrm{H}_{8} \mathrm{O}_{4}$ & 1.60 & 0.80 & 0 & 1.34 & 0.46 & -0.42 & 1.47 & 0.46 & -0.54 \\
Malonic acid & Diacids & $\mathrm{C}_{3} \mathrm{H}_{4} \mathrm{O}_{4}$ & 1.33 & 1.33 & 1.33 & 1.24 & 1.12 & 1 & 1.39 & 0.91 & 0.43 \\
Pimelic acid & Diacids & $\mathrm{C}_{7} \mathrm{H}_{12} \mathrm{O}_{4}$ & 1.71 & 0.57 & -0.57 & 1.30 & 0.34 & -0.62 & 1.56 & 0.31 & -0.94 \\
Succinic acid & Diacids & $\mathrm{C}_{4} \mathrm{H}_{6} \mathrm{O}_{4}$ & 1.50 & 1.00 & 0.50 & 1.57 & 0.51 & -0.55 & 1.56 & 0.46 & -0.65 \\
\hline Sucrose & Alcohols & $\mathrm{C}_{12} \mathrm{H}_{22} \mathrm{O}_{11}$ & 1.83 & 0.92 & 0 & 1.86 & 0.6 & -0.66 & 1.60 & 0.55 & -0.50 \\
Xylitol & Alcohols & $\mathrm{C}_{5} \mathrm{H}_{12} \mathrm{O}_{5}$ & 2.40 & 1.00 & -0.40 & 1.74 & 0.68 & -0.38 & 1.87 & 0.63 & -0.60 \\
\hline
\end{tabular}



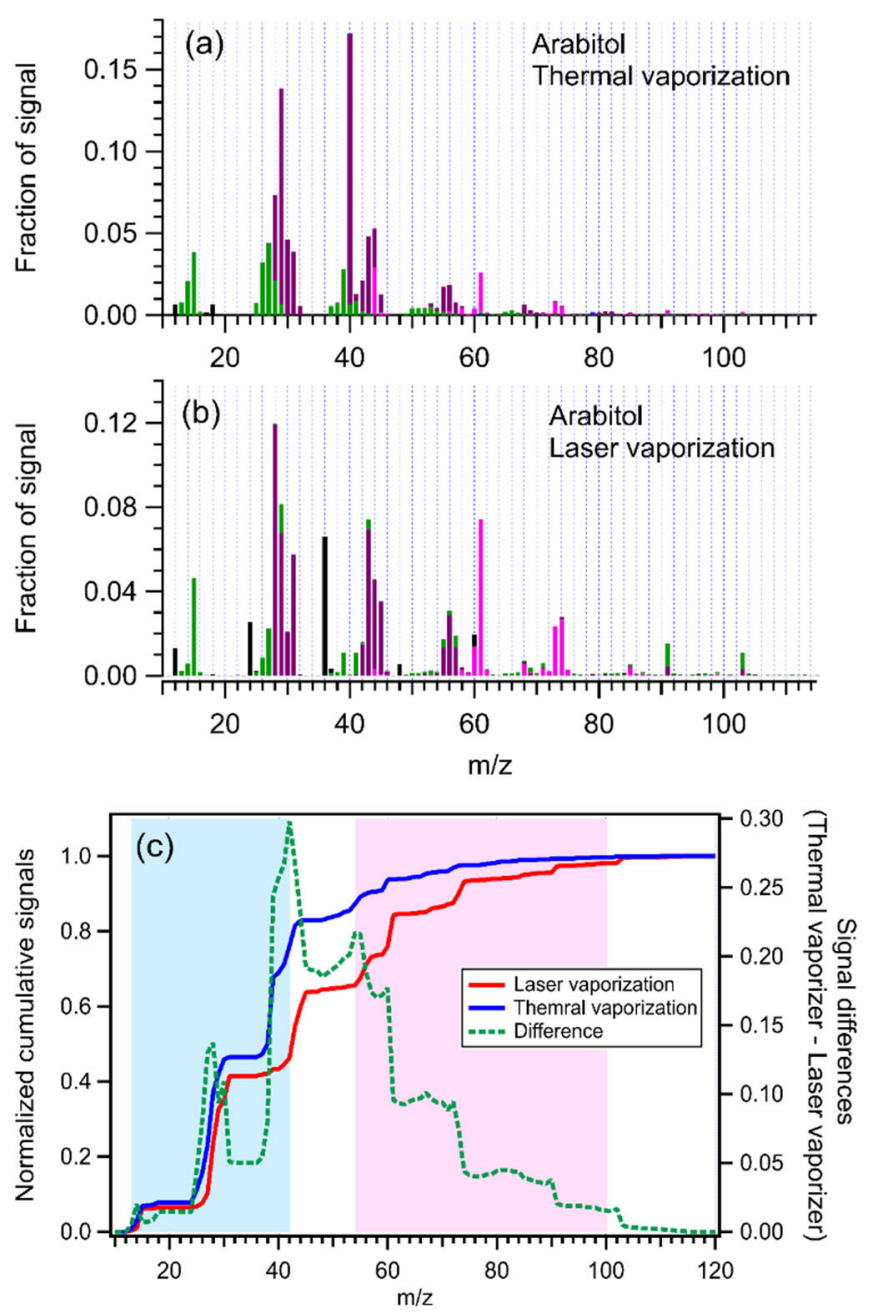

Figure S1. Mass spectra of arabitol, measured by the SP-AMS 1 using the tungsten (a) and laser (b) vaporization schemes. (c) Normalized cumulative histogram of mass-to-charge ratios for the oxygenated organic compounds measured by the SP-AMS 1 . The blue area indicates that the thermal vaporization scheme tends to provide organic fragments with smaller $\mathrm{m} / \mathrm{z}$, whereas the red area indicates that the laser vaporization scheme tends to give organic fragments with larger $\mathrm{m} / \mathrm{z}$. 

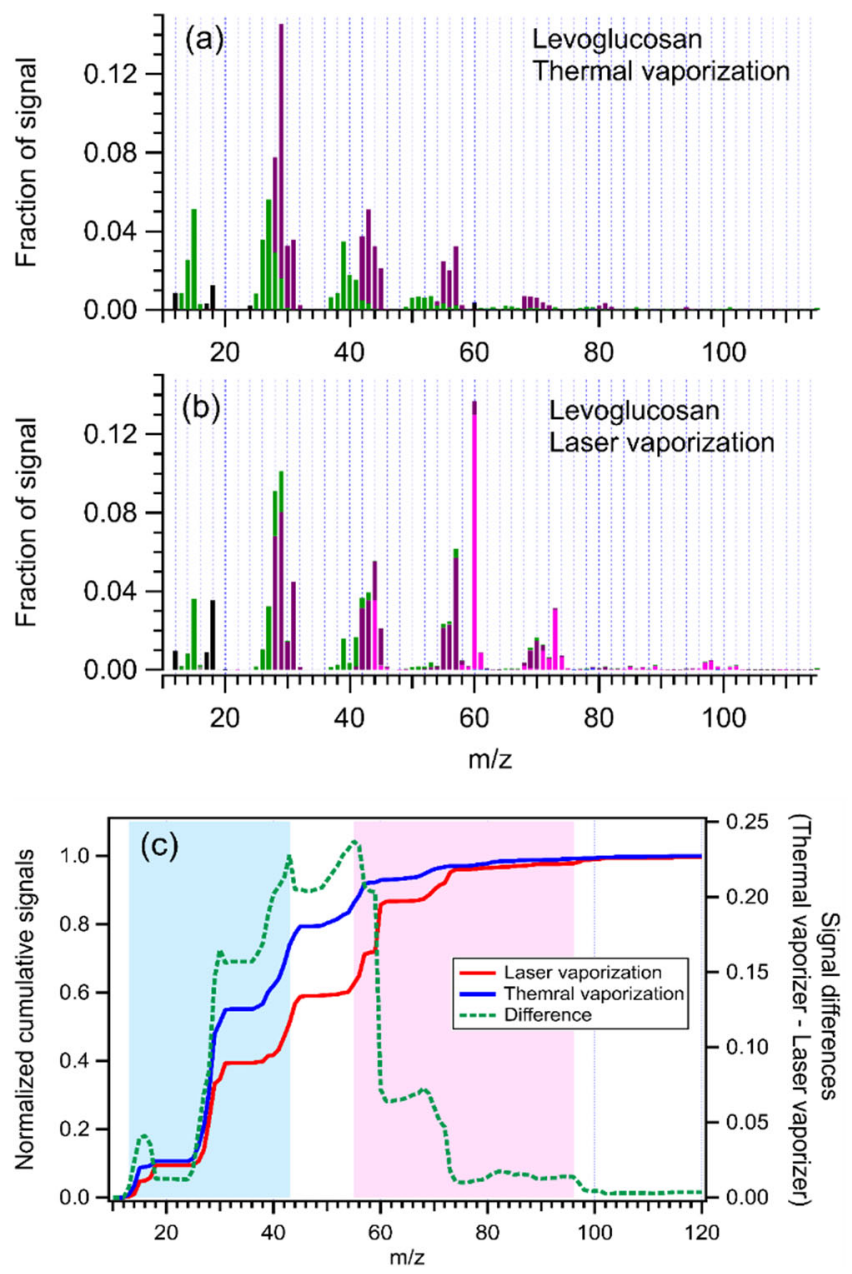

Figure S2. Mass spectra of levoglucosan, measured by the SP-AMS 1 using the tungsten (a) and laser (b) vaporization schemes. (c) Normalized cumulative histogram of mass-to-charge ratios for the oxygenated organic compounds measured by the SP-AMS 1. The blue area indicates that the thermal vaporization scheme tends to provide organic fragments with smaller $\mathrm{m} / \mathrm{z}$, whereas the red area indicates that the laser vaporization scheme tends to give organic fragments with larger $\mathrm{m} / \mathrm{z}$. 

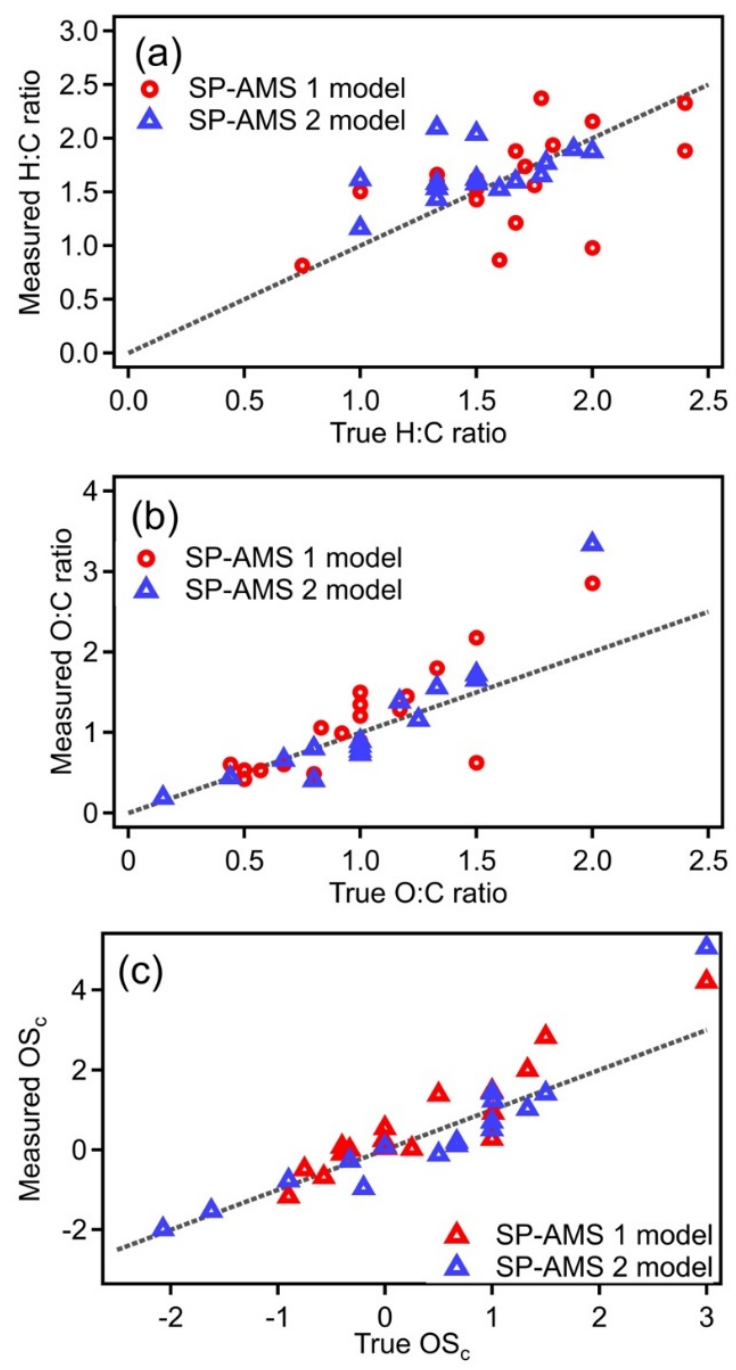

Figure S3. Comparisons between the measured and true values of H:C, O:C, and OSc determined by the two SPAMS using the laser vaporization scheme. The I-Asp method was used for the elemental analysis. Red circles and blue triangles represent data measured by SP-AMS 1 and 2, respectively. The dashed lines represent 1:1 line. 

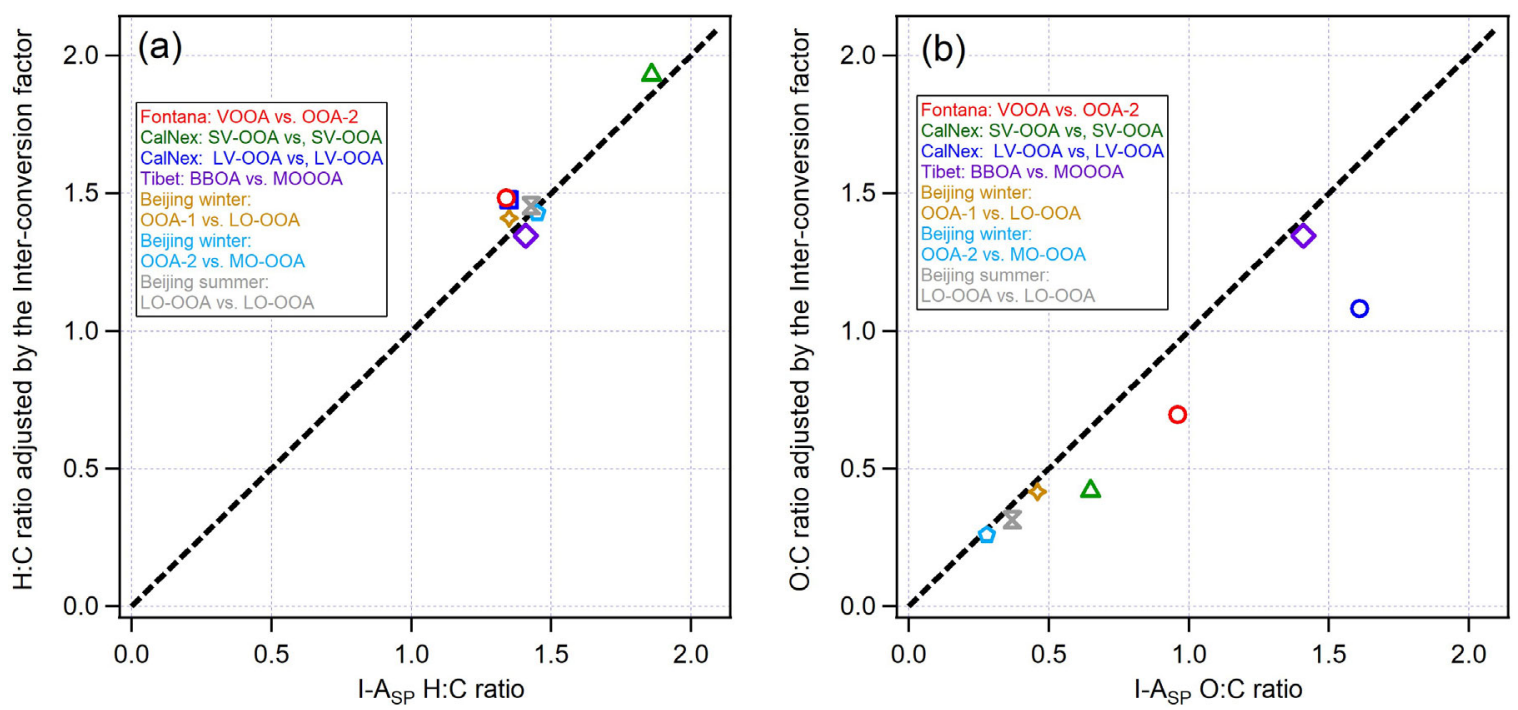

Figure S4. Comparison of elemental ratios determined by the I- $\mathrm{A}_{\mathrm{sp}}$ method and the I-A method with the interconversion factors applied. 

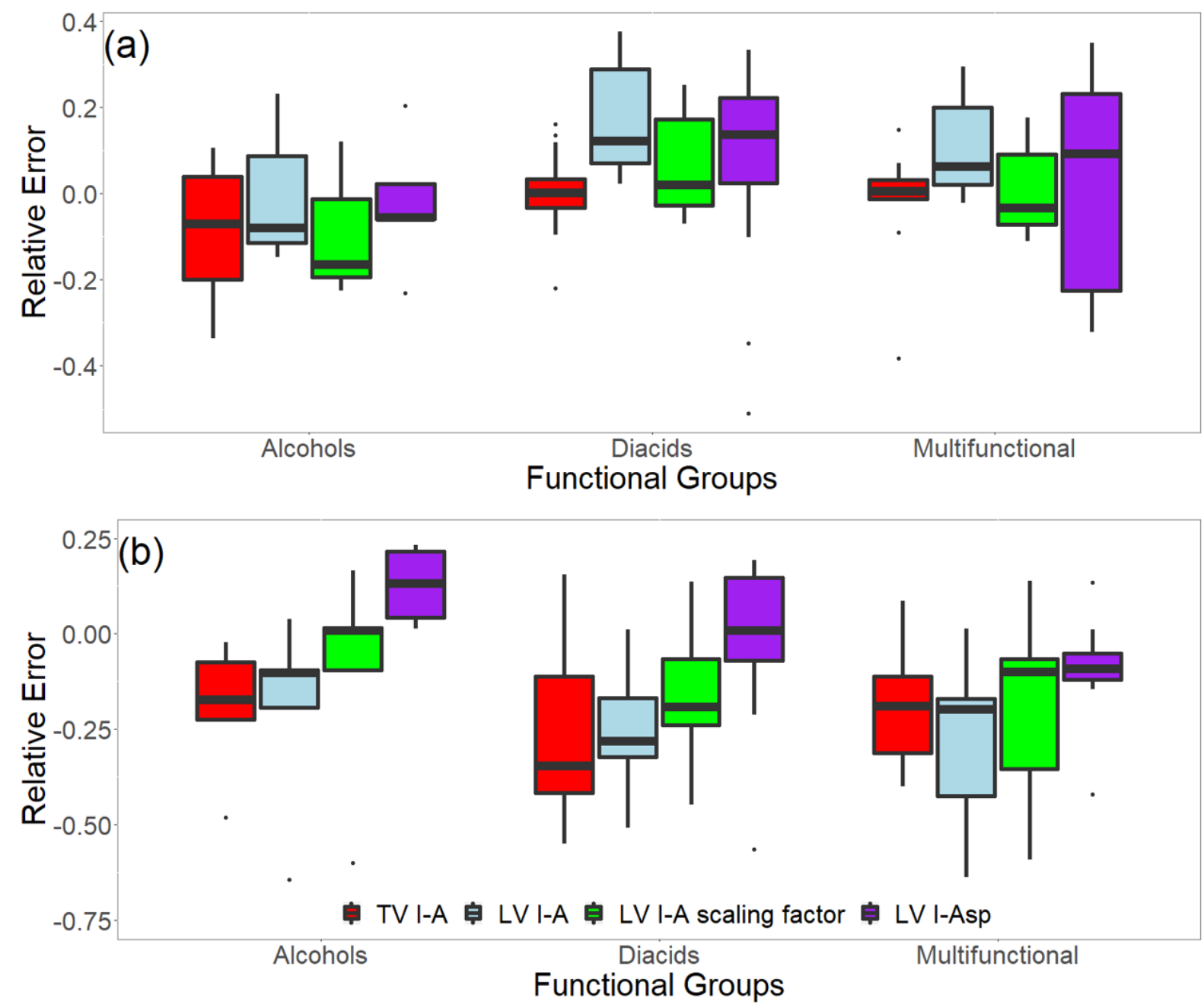

Figure S5. Relative error of H:C (a) and O:C (b) ratios from SP-AMS 1 and 2 calculated with LV and TV methods. TV I-A (red), LV I-A(blue), LV I-A $\mathrm{A}_{\mathrm{sp}}$ (green) and LV I-A scaling factor (purple) are included in the comparison. 


\section{Reference}

Chen, C. L., Chen, S., Russell, L. M., Liu, J., Price, D. J., Betha, R., Sanchez, K. J., Lee, A. K. Y., Williams, L., Collier, S. C., Zhang, Q., Kumar, A., Kleeman, M. J., Zhang, X., and Cappa, C. D.: Organic Aerosol Particle Chemical Properties Associated With Residential Burning and Fog in Wintertime San Joaquin Valley (Fresno) and With Vehicle and Firework Emissions in Summertime South Coast Air Basin (Fontana), J. Geophys. Res.Atmos., 123, 10,707-710,731, 2018.

Lee, A. K. Y., Chen, C. L., Liu, J., Price, D. J., Betha, R., Russell, L. M., Zhang, X., and Cappa, C. D.: Formation of secondary organic aerosol coating on black carbon particles near vehicular emissions, Atmos. Chem. Phys., 17, 15055-15067, 2017.

Massoli, P., Onasch, T. B., Cappa, C. D., Nuamaan, I., Hakala, J., Hayden, K., Li, S.-M., Sueper, D. T., Bates, T. S., Quinn, P. K., Jayne, J. T., and Worsnop, D. R.: Characterization of black carbon-containing particles from soot particle aerosol mass spectrometer measurements on the R/VAtlantisduring CalNex 2010, J. Geophys. Res.-Atmos., 120, 2575-2593, 2015.

Wang, J., Liu, D., Ge, X., Wu, Y., Shen, F., Chen, M., Zhao, J., Xie, C., Wang, Q., Xu, W., Zhang, J., Hu, J., Allan, J., Joshi, R., Fu, P., Coe, H., and Sun, Y.: Characterization of black carbon-containing fine particles in Beijing during wintertime, Atmos. Chem. Phys., 19, 447-458, 2019.

Wang, J., Zhang, Q., Chen, M., Collier, S., Zhou, S., Ge, X., Xu, J., Shi, J., Xie, C., Hu, J., Ge, S., Sun, Y., and Coe, H.: First Chemical Characterization of Refractory Black Carbon Aerosols and Associated Coatings over the Tibetan Plateau (4730 m a.s.l), Environ. Sci. Technol., 51, 14072-14082, 2017.

Xie, C., Xu, W., Wang, J., Liu, D., Ge, X., Zhang, Q., Wang, Q., Du, W., Zhao, J., Zhou, W., Li, J., Fu, P., Wang, Z., Worsnop, D., and Sun, Y.: Light absorption enhancement of black carbon in urban Beijing in summer, Atmos. Environ., 213, 499-504, 2019a.

Xie, C., Xu, W., Wang, J., Wang, Q., Liu, D., Tang, G., Chen, P., Du, W., Zhao, J., Zhang, Y., Zhou, W., Han, T., Bian, Q., Li, J., Fu, P., Wang, Z., Ge, X., Allan, J., Coe, H., and Sun, Y.: Vertical characterization of aerosol optical properties and brown carbon in winter in urban Beijing, China, Atmos. Chem. Phys., 19, 165-179, 2019b.

Xu, J., Zhang, Q., Shi, J., Ge, X., Xie, C., Wang, J., Kang, S., Zhang, R., and Wang, Y.: Chemical characteristics of submicron particles at the central Tibetan Plateau: insights from aerosol mass spectrometry, Atmos. Chem. Phys., 18, 427-443, 2018.

Xu, W., Xie, C., Karnezi, E., Zhang, Q., Wang, J., Pandis, S. N., Ge, X., Zhang, J., An, J., Wang, Q., Zhao, J., Du, W., Qiu, Y., Zhou, W., He, Y., Li, Y., Li, J., Fu, P., Wang, Z., Worsnop, D. R., and Sun, Y.: Summertime aerosol volatility measurements in Beijing, China, Atmos. Chem. Phys., 19, 10205-10216, 2019. 